\title{
DETECTION OF POINTS OF INTEREST FOR GEODESIC CONTOURS: APPLICATION ON ROAD IMAGES FOR CRACK DETECTION
}

\author{
Sylvie CHAMBON \\ Institut Français des Sciences et Technologies des Transports, de l'Aménagement et des Réseaux (IFSTTAR), France \\ chambon@ifsttar.fr
}

Keywords: Detection, segmentation, thin structures, road cracks, point of interest, geodesic contour, auto-correlation.

\begin{abstract}
A new algorithm of automatic extraction of thin structures in textured images is introduced, and, more specifically, is applied to detection of road cracks. The method is based on two steps: the first one consists in detecting points of interest inside the thin structure whereas the second step connects the points with a geodesic contour process. The main contribution of this work is the study of automatic detection of points of interest inside thin structures in a high-textured background. The results are compared with a Markovian segmentation.
\end{abstract}

\section{INTRODUCTION}

This work concerns the detection of road cracks. The threshold-based methods are simple but the results contain a lot of false detections (Koutsopoulos and Downey, 1993). The methods based on morphology allow to reduce false detections but strongly depend on the parameter settings (Tanaka and Uematsu, 1998). The learningbased methods alleviate the problems of the two first categories (Oliveira and Correia, 2008) but they need a learning phase which is not well appropriate to the application. The filtering methods are the most recent ones (Oliveira and Correia, 2009). In (Chambon et al., 2010), we have introduced a segmentation based on a wavelet decomposition and a Markovian modelling. To have a dense detection with less false detections, we introduce a Geodesic Contour-based method with initial automatic detection of Points Of Interest (GCPOID) inside the thin structure. We present, first the data set, second, the method GCPOID, and, the results and conclusions.

\section{DATA SET}

Evaluation is done on 14 synthetic images and 42 real ones. The synthetic images were built using, for 8 , a random dot texture and, for 6 , real images without cracks ( 2 with a static camera and 4 with a camera embedded on a vehicle and controlled lights). The cracks were randomly added, with a random shape and gray-levels (Figure 1). For real images, estimate a reference segmentation is more complicated but the images are more realistic than the synthetic ones. 4 experts have manually segmented the images and the results are merged (Chambon et al., 2010). On the 42 real images, 17 were acquired using a static camera and 25 using a dynamic system.

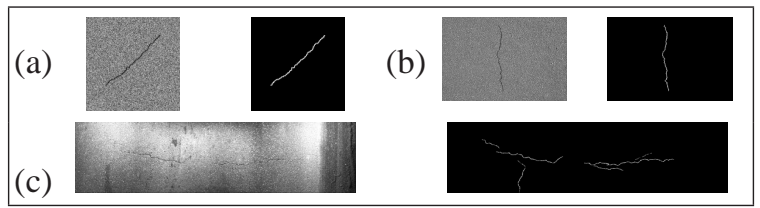

Figure 1: Tested images - one synthetic image (a), one real image with a simulated default (b) and one real image (c).

\section{DESCRIPTION OF GCPOID}

Existing methods have obtained quite good results, particularly, with multiscale analysis (Chambon et al., 2010). However, the results still present a lot of false detections and incomplete detection of the cracks, see Figure 2. Another way to deal with this problem is to consider a sparse but reliable detection and, then, to propagate this detection to obtain a dense detection. In consequence, we propose a Geodesic Contour-based method initialized by 
Points Of Interest Detection (GCPOID), an algorithm in 2-steps : (1) Detection of POIs (inside the cracks), (2) Propagation of the initial detection and the goal of this paper is to propose a detector of POIs efficient for extracting thin structures in textured environment.

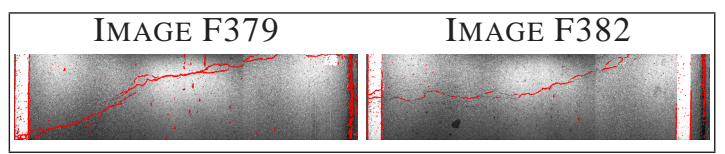

Figure 2: Results obtained with Markovian Modelling (MM) (Chambon et al., 2010) (in red) - This method is based on a first step with adaptive filtering and a refinement step with a Markov model-based segmentation.

Detection of POIs for road cracks - We suppose that points inside the cracks are the POIs in the image because the crack is a significant element of the image, and, moreover, it is a high perturbation in the road texture. Some existing detectors are based on grey level, first derivatives of the image (Harris and Stephens, 1988) or second derivatives (Lowe, 2004). In this work, the most adapted detectors seem to be: Harris operator and grey-level auto-correlation (noted AC) (Moravec, 1977). As an important point of this study, we detail and comment the auto-correlation detector whose principle is:

The grey level of a point of interest must be low correlated to the grey levels of its neighbors:

$$
R\left(\mathbf{p}_{i, j}\right)=\underset{(k, l) \in V(i, j)}{\text { fusion }} M\left(\mathbf{f}_{i j}, \mathbf{f}_{k l}\right),
$$

$R$ depends on $\mathbf{f}_{i j}$ and $\mathbf{f}_{k l}$, the two vectors that contain, respectively, the grey levels of the studied pixel $\mathbf{p}_{i, j}$, and a neighbor pixel $\mathbf{p}_{k, l}$ and their respective neighborhoods, $M$ corresponds to a similarity criterion (correlation measure), "fusion" is an operator to merge the correlation scores in the neighborhood $V(i, j)$ of the studied pixel. In consequence, we have to determined: (V), the size of the neighborhood, $V(i, j),(\mathbf{S})$, the correlation measure and the size of the correlation window $\left(\mathbf{f}_{i}\right)$ and (parameter $\left.\mathbf{F}\right)$, the fusion operator.

Parameter V - For POIs located inside fine cracks on road images, it seems natural and appropriate to take a $3 \times 3$ square area centered on the studied pixel for the size of $V(i, j)$. A different size can be used in an other kind of application.

Parameter S - We have studied the influence of different correlation measures based on our work (Chambon and Crouzil, 2003). For reason of space, the experimentation is not described but it reveals the most appropriate measure, the Normalized
Cross Correlation (NCC), a scalar product between the vectors $\mathbf{f}_{i j}$ and $\mathbf{f}_{k l}$ :

$$
\operatorname{NCC}\left(\mathbf{f}_{i j}, \mathbf{f}_{k l}\right)=\frac{\mathbf{f}_{i j} \cdot \mathbf{f}_{k l}}{\left\|\mathbf{f}_{i j}\right\|\left\|\mathbf{f}_{k l}\right\|} .
$$

The Zero mean NCC (ZNCC) has been used but this centered version of NCC does not afford discriminative values for $R\left(\mathbf{p}_{i, j}\right)$.

Parameter F - Three different kinds of fusion have been proposed:

(1) MAX - When the POIs correspond to the highest dissimilarity in the neighbors, equation (1) becomes:

$$
R\left(\mathbf{p}_{i, j}\right)=\max _{(k, l) \in V(i, j)} M\left(\mathbf{f}_{i j}, \mathbf{f}_{k l}\right) .
$$

(2) MAX-MIN - We suppose that a crack POI has the highest local dissimilarity but also the highest local contrast and equation (1) becomes:

$$
\begin{aligned}
R\left(\mathbf{p}_{i, j}\right)= & \max _{(k, l) \in V(i, j)} M\left(\mathbf{f}_{i j}, \mathbf{f}_{k l}\right) \\
& -\min _{\left(k^{\prime}, l^{\prime}\right) \in V(i, j)} M\left(\mathbf{f}_{i j}, \mathbf{f}_{k^{\prime}, l^{\prime}}\right) .
\end{aligned}
$$

(3) ORIEnTATION - We suppose that the contrast should be oriented and equation (4) becomes:

$$
\begin{aligned}
R\left(\mathbf{p}_{i, j}\right)= & \underset{o}{\operatorname{argmax}}\left(\max _{\left(k_{o}, l_{o}\right)} M\left(\mathbf{f}_{i, j}, \mathbf{f}_{k_{o}, l_{o}}\right)\right. \\
& \left.-\min _{\left(k_{\bar{\sigma}}, l_{\bar{\sigma}}\right)} M\left(\mathbf{f}_{i, j}, \mathbf{f}_{k_{\bar{\sigma}}, l_{\bar{\sigma}}}\right)\right),
\end{aligned}
$$

where the orientation $o$ corresponds to one possible orientation over $\left[0 ; \frac{\pi}{4} ; \frac{\pi}{2} ; \frac{3 \pi}{4}\right]$ and $\bar{o}=\pi+o$.

For each detector, the $N_{p}$ first responses (global selection) are chosen for extracting the $N_{p}$ selected POIs. The influence of $N_{p}$ is studied in $\S 4$.

Geodesic contour propagation - The goal is to find a curve that fits an object or a region by iteratively minimizing a cost or energy function, $E$, of the curve $\mathcal{C}$, defined by (Kass et al., 1988):

$$
E(C)=\int_{0}^{L}\left(w_{1}\left\|C^{\prime}(v)\right\|^{2}+w_{2}\left\|C^{\prime \prime}(v)\right\|^{2}+\mathcal{P}(C(v)) d v\right.
$$

where $c(v)$ represents a curve drawn on a $2 \mathrm{D}$ image, $[0, L]$ its domain of definition, and $L$ the length of the curve. The two first terms represent the internal forces (regularity of the curve) based on first and second derivatives with $w_{1}, w_{2}$ the weights of each of these two parts. The third term is the external force, $\mathcal{P}$. These choices can be made :

$\left(S_{1}\right) \mathcal{P}$ based on grey levels: $\mathcal{P}(C(v))=I(v)$; 
$\left(S_{2}\right)$ P based on the gradients: $\mathcal{P}(\mathcal{C}(v)=g(\|\nabla I(v)\|)$ where $g$ is a decreasing function.

When the evolution curve is based on a planar curve evolution, this is a particular case, the geometric contours (Delagnes and Barba, 1995) that are the most efficient in the presence of topology changes. To combine the advantages of both methods (active and geometric contours) geodesic contours (Cohen and Kimmel, 1997) have been introduced. In fact, it is a particular case of active contours where equation (6) becomes:

$$
E(C)=\int_{\omega}\left(\left\|\mathcal{C}^{\prime}(v)\right\|^{2}+\mathcal{P}(\mathcal{C}(v)) d v\right.
$$

In examining the shape of the element to detect, it seems very natural to use the geodesic contours algorithm and shape $\left(S_{1}\right)$ has been chosen for $\mathcal{P}$. Indeed, the energy function associated to the curve depends on the grey levels (for the external force) instead of the gradients (that are not significant in this kind of images that are highly textured). A Fast Marching algorithm is used for the optimisation. Moreover, we suppose that the cracks are not opened. To rely the $N_{p}$ POIs, denoted by $\mathbf{p}_{i}, i \in\left\{1 ; \ldots ; N_{p}\right\}$, we link the points two by two, by pairs of nearest points:

1. $\mathcal{G} \leftarrow\left\{\mathbf{p}_{i}\right\}$

2. $\mathbf{p} \leftarrow \underset{\mathbf{p} \in \mathcal{G}}{\operatorname{argmin}}\left\|\mathbf{p}_{(0,0)}-\mathbf{p}\right\|$

where $\mathbf{p}_{(0,0)}$ is the origin of the image

3. While $\mathcal{G} \neq \emptyset$ do

(a) $\mathcal{G} \leftarrow \mathcal{G}-\{\mathbf{p}\}$

(b) $\mathbf{p}_{2} \leftarrow \underset{\mathbf{p}_{2} \in \mathcal{G}}{\operatorname{argmin}}\left\|\mathbf{p}-\mathbf{p}_{2}\right\|$

(c) estimate $C$ between $\mathbf{p}$ and $\mathbf{p}_{2}$

(d) $\mathbf{p} \leftarrow \mathbf{p}_{2}$

\section{EXPERIMENTAL RESULTS}

Detection of Points of interest - The results, cf. Figure 3, show that for each method, the best results are obtained when $n=10$, and the larger the number of POIs, the larger the errors. The maximal percentage of detections is always found with a window of size $3 \times 3$. Moreover, auto-correlation always gives better results than Harris, and, the best operator fusion is the maximum. The orientation fusion leads to results similar to those of the maximum. For 38 images (over the 55 tested), we obtained from $80 \%$ to $100 \%$ of points that are well detected inside the cracks. There is no image (over the 55), with a $3 \times 3$ window size, where Harris is the best.

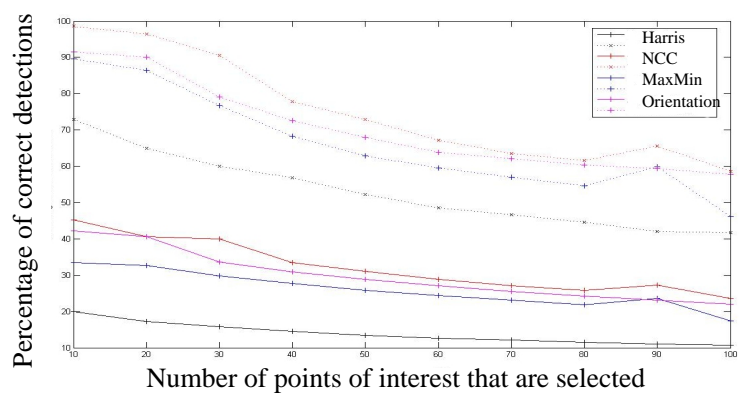

Figure 3: Percentage of POIs detected inside cracks - The mean (solid line) and the maximum (dotted line) percentages are computed with all the results obtained with the 55 images.

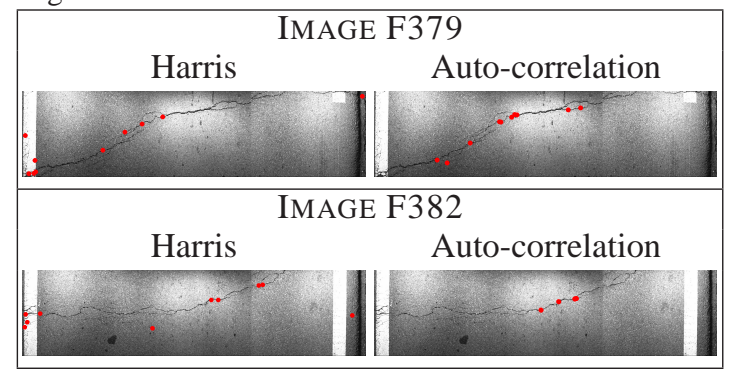

Figure 4: Detection of POIs (in red) in road images - The left results are obtained with the detector of Harris whereas the right, with the auto-correlation (AC) operator. For AC, there is no errors, i.e. no POIs detected outside the cracks.

Crack detection - These two methods are compared: the proposed method GCPOID and the previous method based on Markovian Modelling and denoted by MM (Chambon et al., 2010). These criteria are used in order to compare the results: (1) the percentage of correct matches (TP), (2) the percentage of false negatives (FN) which corresponds to pixels that are not detected as cracks whereas they are inside a crack, (3) the similarity coefficient or Dice similarity, DICE, defined by: $\frac{2 T P}{F N+T P+P}$, where $P$ represents the number of pixels that have been detected as crack pixels. This criterion illustrates the proportion of correct detections compared to the whole detection and it is important for our application because we want to have an accurate and complete detection of the defaults. The higher the Dice (with maximum 1), the better the result.

In the results of GCPOID, for all the images with a percentage of correct detections of POIs of $100 \%$ (27), there is very few false detections. Unfortunately, the detection of the crack is incomplete. For the images with more than $80 \%$ of correct detections inside the cracks, the results are also interesting, i.e better than our initial method MM, cf. figure 5 .

In Figure 6, a global analysis over all the tested images is given. It highlights these two facts: 


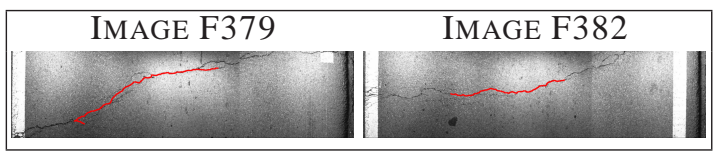

Figure 5: Detection of road cracks with GCPOID - The POIs, obtained with AC (NCC), are linked in red with geodesic contours. Detection is incomplete but with very few false detections when there is no wrong POI.

(1) GCPOID outperforms MM for the percentage of correct matches (TP) ;

(2) On the contrary, MM obtains lower percentages of false negatives (FN) than with GCPOID.

This is why the mean Dice similarity is better with MM, 0.54, than with GCPOID, 0.46. However, the result with GCPOID is reliable: the mean of the correct detections reached $74.43 \%$ (MM obtained only $50.89 \%$ ). In consequence, we can consider that we have reached our first goal: to obtain very few false negatives. The second aim (complete detection) can be obtained if we try to propagate the results of the detection at the extremities of the crack detection, see image F382 in Figure 5.

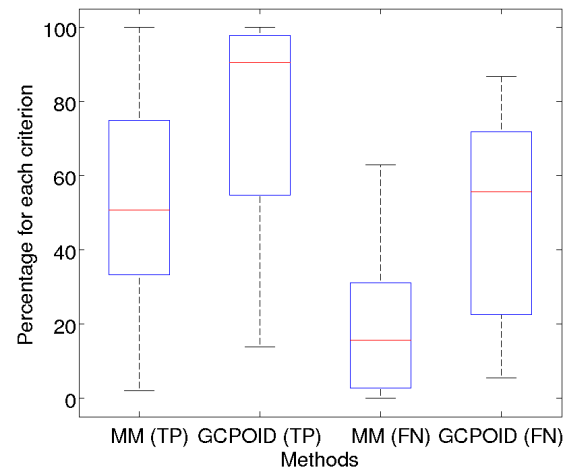

Figure 6: Results obtained with GCPOID and MM - These results are represented by boxes with one box for each criteria (TP, true positives, and FN, false negatives) and each method. The boxes have lines at the lower quartile, the minimum, the median, the maximum and the upper quartile. These results show that the median of the percentages of correct detections is highly improved with GCPOID, because it becomes $90.54 \%$ against $50.68 \%$ for $\mathrm{MM}$ whereas the percentage of false negatives for GCPOID is higher (the median is $55.63 \%$ ) than for MM (the median is $15.68 \%$ ).

\section{CONCLUSIONS}

We introduced a new method of road crack detection based on two steps: automatic detection of POIs followed by the estimation of geodesic contours. The study reveals the most appropriate detector of POIs (by introducing the principle and the pa- rameter settings): auto-correlation detector with normalised cross correlation and fusion with the maximum. Moreover, we demonstrate the interest of using geodesic contours for the final detection by comparing to a Markovian segmentation.

Our future work will focus on how adding more POIs (to have a complete/dense detection), with the combination of prefiltered images with autocorrelation detection, and how removing wrong POIs, by adding some constraints (based on the variation of grey levels in the neighborhood or on the shape of the curves determined by each pair of POIs). For the geodesic contours, we will propose a more realistic algorithm for connecting the POIs (possibility to connect one point with numerous others, to remove a point if the energy is low with all the paths passing through this point).

\section{REFERENCES}

Chambon, S. and Crouzil, A. (2003). Dense matching using correlation: new measures that are robust near occlusions. In BMVC, volume 1, pages 143-152.

Chambon, S., Gourraud, C., Moliard, J., and Nicolle, P. (2010). Road crack extraction with adapted filtering and markov model-based segmentation - introduction and validation. In VISAPP.

Cohen, L. and Kimmel, R. (1997). Global minimum for active contour models: A minimal path approach. IJCV, 24(1):57-78.

Delagnes, P. and Barba, D. (1995). A markov random field for rectilinear structure extraction in pavement distress image analysis. In ICIP, volume 1, pages 446-449.

Harris, C. and Stephens, M. (1988). A combined corner and edge detector. In Alvey Vision Conf., pages 147-151.

Kass, M., Witkin, A., and Terzopoulos, D. (1988). Snakes : Active contour models. IJCV , 1(4):321-331.

Koutsopoulos, H. and Downey, A. (1993). Primitive-based classification of pavement cracking images. Journal of Transportation Engineering, 119(3):402-418.

Lowe, D. (2004). Distinctive image features from scaleinvariant keypoints. IJCV, 60(2):91-110.

Moravec, H. (1977). Toward automatic visual obstacle avoidance. In IJCAI, volume 2, page 584 .

Oliveira, H. and Correia, P. (2008). Supervised strategies for cracks detection in images of road pavement flexible surfaces. In EUSIPCO.

Oliveira, H. and Correia, P. (2009). Automatic road crack segmentation using entropy and image dynamic thresholding. In EUSIPCO.

Tanaka, N. and Uematsu, K. (1998). A crack detection method in road surface images using morphology. In Machine Vision Applications, pages 154-157. 